\title{
Erhaltenen Musikinstrumente aus der Pfarrkirche Mariä Himmelfahrt in Kvasice im Hinblick auf museale Sammlungen in Wien und Brünn
}

\section{Preserved Musical Instruments from the Church of the Assumption of Our Lady in Kvasice in Museum Collections in Vienna and Brno}

Miroslav Lukeš / 31943@mail.muni.cz

Department of Musicology, Faculty of Arts, Masaryk University, Brno, CZ

\begin{abstract}
This study traces the history and use of the preserved musical instruments from the organloft of the church of the Assumption of Our Lady in Kvasice. Currently, they are placed in the museum collections Gesellschaft der Musikfreunde in Vienna and at the Department of the History of Music of Moravian Museum in Brno.

Theodora Straková not only wrote a report about this rare inventory of music in her study Kvasický inventár z r. 1757 (Kvasice Inventory from 1757), but also presented other preserved inventories of church property. She provided basic information about the instruments from the loft of the church, which gave rise to the creation of this study. In this study, the fate of the musical instruments coming from the loft of the church of the Assumption of Our Lady in Kvasice is mapped. After a period of time when they were not used during the church activities, Dr. Emanuel Proskowetz jr. (1849-1944) bought them from the priest F. Ignác Němec in Kvasice in 1905. Dr. Proskowetz jr. was a breeder, economist, water management expert and the president of the management of sugar factories. Apart from this, he was an excellent organist and music lover. He donated some of the instruments to the collections of Gesellschaft der Musikfreunde in Vienna later in life. Other instruments are part of the collections of the Department of the History of Music of Moravian Museum in Brno. The museum obtained them via Adolf Straka (1883-1960)the director of the loft of the church in Kvasice - either as a gift or as a part of his inheritance. The preserved instruments were compared with the entries in the church inventories that are part of the Parish Office in Kvasice and are stored in the State District Archives of Kroměŕžz.
\end{abstract}

\section{Keywords}

Moravia, Kvasice, music collection, musical instruments, organ, violin, Rottal, Proskowetz, Straka 
„... die erhaltenen Musikinstrumente illustrieren das Bild der musikalischen

Entwicklung in vergangenen Jahrhunderten." Theodora Straková - Kvasický inventár z r. 1757.

Die Rekonstruktion eines komplexen Bildes, das sowohl Musikalien als auch Musikinstrumente von einem Chor in Rücksicht nehmen würde, ist leider immer eine schwere Aufgabe und ist nur in wenigen Fällen möglich. ${ }^{2}$ Wenn dann beides aus einer Lokalität erhalten ist, und dazu man noch über sekundäre Quelle (wie z. B. Inventare) verfügt, kann ein lebendiges, vielschichtiges Zeugnis der lokalen Musikgeschichte dargestellt werden. Die oben geschilderte Situation betrifft die mährische Stadt Kvasice (Kwassitz), die sich 10 Kilometer südöstlich von der Stadt Kroměříž (Kremsier) befindet. Dank der Wohltätigkeitsaktivitäten des Herrn von Rottal, insbesondere von Joachim Adam Graf von Rottal, ${ }^{3}$ der in den Jahren 1730-1740 eine neue Kirche Mariä Himmelfahrt bauen ließ, kann heute die musikalische Aktivität in Kvasice wissenschaftlich untersucht werden. Auf dem Musikchor ist im 18. Jahrhundert eine Musiksammlung entstanden, die heutzutage in der Abteilung der Musikgeschichte des Mährischen Landesmuseums in Brno (Brünn) aufbewahrt wird. ${ }^{4}$

In der Abhandlung Kvasický inventár z r. $1757^{5}$ berichtete Theodora Straková nicht nur über das wertvolle Noteninventar, sondern berücksichtigte sie auch die weiteren erhaltenen Inventare über Kirchenvermögen. Somit bot sie die grundlegenden Informationen über den Zustand der Musikinstrumente an. Dies war ein Impuls zur Erstellung dieser Abhandlung, in deren Rahmen das Schicksal der Musikinstrumente, die zur Kirche Mariä Himmelfahrt gehörten, näher eingegangen wird. Das betrifft die für den Betrieb nicht mehr verwendbare Instrumente, die vom damaligen Pfarrer P. Ignatz Němec im Jahre 1905 Dr. Emanuel Proskowetz jun. ${ }^{6}$ abkaufte. Einige der Instrumente schenkte er später in die Sammlungen der Gesellschaft der Musikfreunde (GMF) in Wien. Andere Instrumente gehören heutzutage zu den Sammlungen der Abteilung der Musikgeschichte des Mährischen Landesmuseums (ODH MZM) in Brünn, in die sie dank des Kvasicer Chordirektors Adolf Straka (1883-1960) als Geschenk oder als Bestandteil seines Nachlasses kamen. Deshalb können die erhaltenen Instrumente mit den Einträgen in den Kircheninventaren verglichen werden, die zum Beständen der Pfarrei in Kvasice gehören

1 STRAKOVÁ, Theodora. Kvasický inventář z r. 1757. In Časopis Moravského musea. roč. 38, Brno 1953, S. 105.

2 Diese Studie wurde im Rahmen des Projekts von der Grant-Agentur der Tschechischen Republik „Die Musikinventare der Frühneuzeit in den Böhmischen Ländern“ (GA16-17615S) vorbereitet.

3 Joachim Adam Graf von Rottal gehörte zum Zweig der Familie aus Napajedla (Napajedl) und mit Angehörigen des Zweigs der Familie aus Holešov Franz Anton Graf von Rottal sind sie verwandt, weil sie einen gemeinsamen Vorfahren den Urgroßvater George Julius hatten (siehe Abbildung 1).

4 Ausführlich beschäftigt sich der Autor dieser Abhandlung mit der Problematik in Rahmen seiner Dissertation namens Thematisches Verzeichnis der Musiksammlung der Mariä-Himmelfahrt-Kirche mit dem Fokus auf das Schaffen von Václav Kalous.

5 STRAKOVÁ, Theodora. Kvasický inventář z r. 1757. In Časopis Moravského musea. roč. 38, Brno 1953.

6 Dr. Emanuel Proskowetz jun. (1849-1944) war Züchter, Wirtschaftswissenschaftler, Experte für Wasserwirtschaft und der Verwaltungspräsident der Zuckerfabriken. Darüber hinaus war er ein hervorragender Organist und Musikliebhaber. 
und sich derzeit in der SOkA in Kroměříž unter Sign. Bj-20 befinden. Zuerst wird jedoch die Aufmerksamkeit an die Geschichte der Orgel in Kvasice gerichtet.

\section{Orgel auf dem Chor in Kvasice}

Auf dem Chor ließ der Erbauer und Stifter der neuen Kirche Mariä Himmelfahrt Joachim Adam Graf von Rottal eine neue Orgel bauen. In den Jahren 1739-1740 wurde eine zweiteilige Orgel mit zwei Manualen und einem Pedal mit kurzer Oktave vom Orgelbauer Adam König gebaut. Sie befand sich in zwei reich geschnitzten und prachtvoll vergoldeten barocken Schränken. Der dritte Teil der Orgel - Positiv, in dem sich die Pfeifen des zweiten Manuals befanden - stand mitten auf dem Chorgeländer, wobei der Spieltisch vor dem Positiv stand. Die Orgel wurde 160 Jahren gespielt, bis sie durch ein neues Instrument ersetzt wurden. ${ }^{7}$

Wie Jiří Sehnal in seiner Monographie Barokni varhanářství na Moravě berichtet, wurden an den Reparaturarbeiten des Instruments seien mehrere Orgelbauer beteiligt. Im Jahre 1767 wurde die Orgel und das Positiv für 180 fl. von Christian Wieser, Orgelbauer in Kroměříž repariert. ${ }^{8}$ Später im Jahre 1795 führte den Orgelumbau Johann Hasse, Orgelbauer in Olomouc (Olmütz) und Velká Bystřice (Groß Wisternitz) für 420 fl. durch und später noch einmal im Jahre 1802 für 30 fl. ${ }^{9}$ Das Instrument ist teilweise erhalten geblieben, weil der Orgeleschrank 1900 für den Orgelbau in der St. Margarethen Kirche in Jaroměřice nad Rokytnou (Jarmeritz) verwendet wurde. ${ }^{10}$ Den besten Beweis dafür stellt ein Vergleich dar - verglichen sei eine Kopie des für Kvasice vorgeschlagenen Orgelentwurfs von Adam König, der in der Mitte vom Graf Adam von Rottal signiert wurde (siehe Abbildung 2) und ein Foto, das den aktuellen Stand der Orgel in Jaroměřice illustriert (siehe Abbildung 3).

Straková behauptet, dass - laut der Mitteilung ihres Schwiegervaters Adolf Straka im Jahre 1905 - der damalige Pfarrer in Kvasice P. Ignác Němec die Musikinstrumente aus der Kirche dem Herrn Proskowetz geschenkt oder verkauft haben solle. ${ }^{11}$ Proskowetz erhielt u. a. den Spieltisch von der Königs Orgel, offenbar als eine Danksagung für eine beträchtliche Summe, die er für neue Orgel spendete. Was war aber das Schicksal des Spieltisches von Königs Orgel, die im Jahre 1900 durch ein neues Instrument ersetzt wurde? In den Sammlungen der Gesellschaft der Musikfreunde in Wien befindet sich ein Spieltisch mit zwei Manualen und 11 Registern (Sign. I. N. 359). Gemäß den Angaben im Jahresbericht der Gesellschaft vom Jahre 1917/1918 spendete dieses Ausstellungsstück

7 SOkA Kroměřǐž. Farni fond Kvasice. sign. Bj-20.

8 SEHNAL, Jiří. Barokni varhanářstvi na Moravě. Díl. 1. Varhanáři. Muzejní a vlastivědná společnost v Brně, 2003. S. 139-140.

9 Ebd. S. 55.

10 Ebd. S. 75.

11 STRAKOVÁ, Theodora. Kvasický inventár z r. 1757. In Časopis Moravského musea. roč. 38, Brno 1953, S. 109. 
zusammen mit zwei anderen gerade E. Proskowetz, der damals Vize-Präsident der Gesellschaft der Musikfreunde war. ${ }^{12}$ In einer Notiz über die Spende ist angegeben: „F. Harbich in Brünn (wahrscheinlich nur von ihm repariert)“. Offensichtlich scheint zu sein, dass das Instrument mit einem Etikett von Brünner Orgelbauer Franz Harbich ${ }^{13}$ versehen ist, ${ }^{14}$ der jedoch den von König gebauten Spieltisch aus Kvasice nur reparierte.

Am Ende des 19. Jahrhunderts fiel die Entscheidung über den Erwerb der neuen Orgel. Die nächste Orgel der Pfarrkirche stammen von der Firma Rieger Krnov (Jägerndorf). Der Entwurf wird ins Jahr 1899 datiert und wurde bereits 1900 als 765. gebaute Orgel der Firma für 8770 Kronen abgeschlossen. Dies ergibt sich aus den Akten im Bestand des Pfarramtes Kvasice, in dem die Korrespondenz zwischen der Firma Rieger und dem ehemaligen Pfarrer P. Ignác Němec bezüglich der Orgeldisposition erhalten ist. ${ }^{15}$ Die Orgel verfügt über 2 Manuale und 23 Register, sowie eine mechanische Traktur.

An der Jahrtausendwende brauchte die Orgel dringend eine komplexe Reparatur, auf die die Gemeinde nicht genügend Mittel hatte, deshalb wurde stattdessen eine Elektroorgel erworben. Diese dritte Orgel in der Kirche stammen von der italienischen Firma Intercontinental Electronics, SpA Mondaino. Es handelt sich um ein digitales Instrument der Marke Viscount mit drei Manualen, dessen Installation Mitte März 2003 stattfand. ${ }^{16}$ Es geht um ein kleines Elektromusikinstrument, was erlaubte, die alte Orgel von Rieger auf dem Chor zu lassen. Deswegen kann im Falle, dass es in der Zukunft die notwendigen Finanzmittel zu sichern gelingt, um die Orgel zu reparieren, sie wieder zum Erklingen bringen.

Interessant ist das Schicksal des Portativs, das aus der älteren, heutzutage der Filialkirche Mariä Himmelfahrt stammt. Das Instrument wurde in die neue Kirche auf dem Platz übertragen und wurde da während des Fronleichnamsfestes verwendet. Im Inventar vom 1752 ist er als Positivum seu parvum organum 5 (quinque) mutationum portabile bezeichnet. ${ }^{17}$

Straková äußert sich darüber folgend: „[...] In diese Sammlung [Gesellschaft der Musikfreunde] wurde später auch das Portativ aus der ursprünglichen Kirche verschickt, die vom Orgelbauer König in Jahren 1739-1740 gebaut wurde. "18 Tatsächlich befindet sich das Instrument in den Sammlungen der Gesellschaft der Musikfreunde (Sign. I. N. 348). Proskowetz schenkte ihn in der Gesellschaft schon in der Saison 1914/1915. ${ }^{19}$ Darüber schreibt

12 Jahresbericht der Direktion für das 106. Vereinsjahr (1917/18) 1. Juli 1917 bis 30. Juni 1918. Erstattet in der ordentlichen Generalversammlung am 19. Dezember 1918. Wien 1918. S. 82.

13 Orgelbauer Franz Harbich - geboren 1780 in Valteřice u Vysokých Žibřidovic (Waltersdorf bei Hohenseibersdorf), tätig in Brno (Brünn), wo er auch 1862 gestorben ist

14 Jahresbericht der Direktion für das 106. Vereinsjahr (1917/18) 1. Juli 1917 bis 30. Juni 1918. Erstattet in der ordentlichen Generalversammlung am 19. Dezember 1918. Wien 1918. S. 82.

15 SOkA Kroměříž. Farni fond Kvasice. sign. Bj-20.

16 DOSOUDIL, Václav. Katolická farnost Kvasice 2003. In Kvasické noviny. Kvasice, 3/2003, S. 4.

17 SOkA Kroměříz. Farni fond Kvasice. sign. Bj-20.

18 STRAKOVÁ, Theodora. Kvasický inventář z r. 1757. In Časopis Moravského musea. roč. 38, Brno 1953, S. 109.

19 Jahresbericht der Direktion für das 103. Vereinsjahr (1914/15) 1. Juli 1914 bis 30. Juni 1915. Erstattet in der ordentlichen Generalversammlung am 16. Dezember 1915. Wien 1915. S. 80.

Jahresbericht der Direktion für das 106. Vereinsjahr (1917/18) 1. Juli 1917 bis 30. Juni 1918. Erstattet in der ordentlichen Generalversammlung am 19. Dezember 1918. Wien 1915. S. 80. 
auch J. Sehnal mit einem Hinweis, dass es um ein Positiv aus der Zeit vom 1750 geht, dass nach dem II. Weltkrieg von sowjetischen Soldaten beschädigt wurde. ${ }^{20}$

\section{Kirchenmusikinstrumente aus Kvasice}

Zur üblichen Ausstattung eines Musikchores im 18. Jahrhundert gehörten, neben der Orgel, hauptsächlich Streichinstrumente, Blech- und Holzblasinstrumente zusammen mit Pauken. Nach der Liste der verfügbaren Inventare ist offensichtlich, dass der Kvasicer Chor für kirchliche Figuralmusik sehr gut ausgestattet war. Die wichtigsten Quellen dazu stellen die Noten- und Musikinstrumenteninventare dar.

Die älteste Auflistung der Musikausstattung aus dieser Kirche wurde im Inventar über Bestandsaufnahme des kirchlichen Eigentums aus dem Jahre 1752 verfasst, ${ }^{21}$ das von dem damaligen Pfarrer P. Mathias Moritz (24. 2. 1711- 3. 3. 1782) in lateinischer Sprache verfasst wurde. An erster Stelle wurde die Orgel mit 12 Registern notiert, dann folgen Musikinstrumente, die aus der Filialkirche der Mariä Himmelfahrt in die neue Kirche mit dem gleichen Patrozinium übertragen worden sind: ein tragbares Positiv mit 5 Registern, Violon (oder Kontrabass), Violoncello samt Bezug, zwei Violinen, ein paar Pauken und vier Posaunen. ${ }^{22}$

Interessante Informationen bringt das Inventar des kirchlichen Eigentums vom Jahre $1788 .^{23}$ Im ersten Kapitel Supellex Musica werden zuerst die sich wiederholende Einträge aus dem Jahre 1752 aufgelistet, danach folgen mit einer unterschiedlichen Hand später eingekaufte Musikinstrumente, und zwar bis zum Jahre 1791. ${ }^{24}$

Als Erstes steht in der Liste eine Violine von dem Cremoner Geigenbauer Nicola Amati, datiert ins 1694. Angesichts der Tatsache, dass Amati zehn Jahre früher (12. 4. 1684) verstorben ist, ${ }^{25}$ konnte das Instrument erst nach seinem Tod gekauft werden, beziehungsweise konnte sich um einen anderen Geigenbauer handeln, der die Amati-Marke verwenden könnte. Weiter folgt eine Violine von Johann Franz Plachta aus Schönbach (Luby u Chebu). Im Buch Češti houslaři berichtet Karel Jalovec über ihn, dass er wahrscheinlich ein Sohn von František Plachta I. war und dass die von ihm gefertigte

20 SEHNAL, Jiří. Barokni varhanářstvi na Moravě. Dil. 2. Varhany. Muzejní a vlastivědná společnost v Brně, 2004. S. 156.

21 SOkA Kroměříz. Farni fond Kvasice. Sign. Bj-20.

22 Organum magnum cum pedali (12 mutationum), Positivum seu parvum organum (5 mutationum) portabile, Violon seu bassus magnus, Violoncello in futerali, Duo fides musica, Unum par tympanorum, Quatuor tuba ex aurichalcina cum requisitis.

23 SOkA Kroměříž. Farni fond Kvasice. Sign. Bj-20.

24 Fides musica - Cremonensis authore Nicolao Amatio 1694 / dtto [fides musica] Joane Franz. Placht / Schönbachü 1769 / alio fides musica Jacobo Stainer authore / dtto alio ignoto authore / Violon seu bassus magnus musicus novus 1789 comparatus / Fides musica duo antiquo 1789 Vienna procurata cum futerali / 1 par Fistularum sie dicto Clarini 1791 comp. / 1 Fagotte novum Vienno comp. 1791.

25 JALOVEC, Karel. Italšti houslaři. Orbis Praha, 1952. S. 45. 
Violinen mittlerer Qualität waren. ${ }^{26}$ Dritte Violine in der Liste sollte ein österreichischer Geigenbauer Jacob Stainer herstellen. In diesem Falle ist das Herstellungsjahr nicht angegeben. Jedoch ist es ziemlich bekannt, dass es sogar noch im 18. Jahrhundert üblich war, dass einige Geigenbauer ihre Instrumente mit Stainer-Etikett auch nach seinem Tod versahen. ${ }^{27}$ Weder in den Sammlungen der GMF in Wien, noch in ODH MZM in Brünn befindet sich ein Musikinstrument von Geigenbauern Amati, Stainer oder Plachta aus der Provenienz Kvasice, demnach ist ihr Schicksal unbekannt. Das dritte Instrument aus Kvasice, das Proskowetz nach Wien widmete, ist eine Bratsche. (Sing. I. N. 345). In der Bemerkung steht: Ein Instrument von schönem, vollem Klang, aber unbequemer Mensur, etwa 100 Jahre alt..$^{28}$

Im Inventar des Kirchenvermögens aus dem Jahre 1845 im Kapitel Musikalischen Instrumenten ist ein Zuwachs an Musikinstrumenten, insbesondere an Blas- und Streichinstrumenten aufgezeichnet worden..$^{29}$ Noch im Jahre 1905 befanden sich auf dem Chor viele Musikinstrumente (17 Violinen, Bratschen, Cello, Kontrabass, Pauken, Fagotte, Hörner, Zugposaunen, Trompeten, Querflöten, Klarinetten und Oboen). ${ }^{30}$

Straková schreibt über die Instrumente der Chor in Kvasice weiter: „Nur ein kleiner Teil wurde Kvasice gelassen und überging gemeinsam mit dem übernommenen Musikinventar aus Proskowetz-Eigentum im Jahr 1945 als staatliches Depositum in das Mährische Landesmuseum in Brno." ${ }^{\text {"I } 1}$ In der Tat ist im Depositar der Abteilung für Musikgeschichte des Mährischen Landesmuseums eine erhebliche Menge der Musikinstrumente ursprünglich aus Kvasice. Zwecks dieser Studie wurden nur diejenigen Instrumente ausgewählt, bei denen es zu vermuten ist, dass sie aus den Kirchenbeständen kommen können. Dagegen waren solche Instrumente ausgeschlossen, die offensichtlich nicht für den Kirchenmusikbetrieb verwendet wurden (Gitarren, Zithern und Walzen-Spieldose). Andere Musikinstrumente aus Provenienz Kvasice greifen auf die Sammlungstätigkeit von Adolf Straka zurück.

Aus der Sammlung von Proskowetz stammt ein Violoncello vom italienischen Geigermacher Sancto Seraphin $^{32}$ (Sign. E 166), das nach dem Jahr 1945 mithilfe Adolf Straka in die Sammlungen der ODH MZM in Brno kam. Überdies kam das Violoncello gewisse Zeit lang zur Geltung, denn es wurde vom Janáčeks-Streichquartett verwendet. Im Depositar des MZM im Schloss Jevišovice (Jaispitz) in der Nähe von Brno ist das Violoncello bis heute bewahrt. Auf seinem Etikett steht: Sanctus Seraphin, Utinensis fecit

26 JALOVEC, Karel. Češti houslaři. Státní nakladatelství krásné literatury, hudby a umění Praha, 1959, S. 89.

27 SENN, Walter - ROY, Karl. Stainer, Jacob. [online]. In Grove Music Online. [Cit. 2017-08-25]. Zugänglich unter: 〈http://www.oxfordmusiconline.com/subscriber/article/grove/music/26528>.

28 Jahresbericht der Direktion für das 106. Vereinsjahr (1917/18) 1. Juli 1917 bis 30. Juni 1918. Erstattet in der ordentlichen Generalversammlung am 19. Dezember 1918. Wien 1918. S. 80.

29 SOkA Kroměříž. Farni fond Kvasice. Sign. Bj-20.

30 STRAKOVÁ, Theodora. Kvasický inventár̆ z r. 1757. In Časopis Moravského musea. roč. 38, Brno, 1953, S. 109 .

31 Ebd. S. 109.

32 Der Geigermacher Sancto Seraphin (wurde 1699 in Udine geboren und im Jahre 1776 in Venedig verstorben. 
Venetiis anno 1707. Ein Problem liegt darin, dass Sanctus Seraphin im Jahre 1699 in Udine geboren wurde. Es ist unmöglich, dass er mit acht Jahren im Stande war, jegliches Musikinstrument herzustellen. Nach den nachgewiesenen Informationen kam er nach Venedig um 1720 und arbeitete zunächst bei anderen Meistern. Seine ersten Etiketten, die seine eigenständige Tätigkeit belegen, stammen erst aus dem Jahre $1725 .{ }^{33}$ Es ist ein weiterer Beweis für die Etikett-Fälschung, wobei seinem Gestalter anscheinend nicht das Geburtsjahr von Seraphin bekannt wurde. Nähere Informationen über das Violoncello stellte Břetislav Vybíral, Mitglied des Janáček-Quartetts und Nachfolger des Gründungsmitglieds Karel Krafka (das Violoncello benutzten beide Musiker) zur Verfügung. ${ }^{34}$ Laut seinen Worten begutachtete das Instrument während einer Konzert-Tour nach London ein Experte für Streichinstrumente von der Firma W. E. Hill \& Sons. Seines Erachtens geht es nicht um ein Instrument aus der Werkstatt von Seraphin, sondern um ein Werk von einem näher nicht bestimmten Geigenbauer aus deutschsprachigem Raum. B. Vybíral hörte später auf, das Violoncello zu spielen, weil es wegen zahlreicher Transporten begann sich abzulösen und häufig repariert werden musste. Das Instrument wurde dann wieder in die Sammlungen der ODH MZM aufgenommen.

Die folgende Auflistung beinhaltet Musikinstrumente aus dem Chor in Kvasice, die in die Sammlungen der ODH MZM von Adolf Straka abgekauft wurden oder ein Bestandteil seines Nachlasses erworbenen nach dem Jahr 1960 waren:

- E 150 Querflöte aus Ebenholz

- $\quad$ E 334 Klarinette aus Buchsbaum Und (Kaspar Tauber), Wien, 1798-1829

- E 335 Klarinette Buchsbaum. Und (G. Keyha), Prag (ca). 1800

- E 336 Klarinette Buchsbaum. B

- E 337 Klarinette Buchsbaum. B

- E 338 Klarinette Buchsbaum. C (W. Hutal), Prag

- E 339 Klarinette Buchsbaum. C, Vienna

- E 340 Oboe Buchsbaum. (Franz Strobach), Karlovy Vary (Karlsbad) Anfang des 19. Jahrhunderts.

- $\quad$ E 341 Oboe Buchsbaum. ALT (R. Paur) Hälfte. 18. Jahrhundert.

- $\quad$ E 342 Oboe Buchsbaum. d, Amore (R. Paur) Hälfte. 18. Jahrhundert.

- $\quad$ E 343 Fagott (Franz Harrach), Wien (ca). 1800

- E 344 Fagott

- E 345 Fagott

- E 346 Fagott

- $\quad$ E 347 Waldhorn (Ferd. Hell), 1830-1841 Brno (Brünn), 1841-1869 Wien

- $\quad$ E 348 Waldhorn (Ferd. Hell), 1830-1841 Brno (Brünn), 1841-1869 Wien

- E 349 Inventionstrompete

- $\quad$ E 394 Fagott (Ferd. Hell, Wien), 1830-1841 Brno (Brünn), 1841-1869 Wien

- $\quad$ E 395 Fagott

33 BEARE, Charles. Seraphin, Sanctus [online]. In Grove Music Online. [cit. 2017-08-25]. Zugänglich unter: <http://www.oxfordmusiconline.com/subscriber/article/grove/music/25445>.

34 Persönliches Gespräch mit Břetislav Vybíral am 25 April. 6. 2017. 
- E 396 Fagott (Huittl Ignatz), Kraslice (Graslitz) um 1800

- E 397 Fagott

- E 398 Fagott (Joseph Ziegler), Wien, 19. Jahrhundert.

- E 399 Bassklarinette (W. Kohlert und Söhne), Kraslice (Graslitz), 1. Hälfte des 20. Jahrhunderts.

- E 400 Bass Tuba (Daniel Fuchs, Wien)

- E 412 Geige (Jakob Kliment), Brno (Brünn), 1846-1893 Geigenbauer

- E 413 Geige (Josef Kliment), Brno (Brünn), 1863-1914, Neffe von Jakob Kliment

- E 414 Geige (Gebr. Placht, Wien-Pest 1824) - der Ursprung in Luby (Schönbach bei Eger)

- E 416 Bratsche (Johann Udalricus Eberle) Prag 1717-1768

- E 417 Viola

- E 419 Violoncello

- E 421 Oboe (Stanzl), Wien

Für Instrumente, bei denen der Hersteller, einschließlich des Zeitraums seiner Tätigkeit bekannt ist, wurde die ungefähre Datierung der Entstehungszeit ergänzt Angesichts dessen, dass manche Musikinstrumente in Sammlungen in Brno weder mit den Angaben über Hersteller noch vom Entstehungsjahr versehen sind, ist es schwierig, die einzelnen Angaben vom Inventar aus Kvasice mit den Instrumenten in Verbindung zu setzen.

\section{Fazit}

Aus den oben genannten Forschungsergebnissen geht hervor, dass eine große Anzahl von Musikinstrumenten aus der Pfarrkirche Mariä Himmelfahrt bis heute erhalten geblieben sind, vor allem dank der Sammeltätigkeit von Emanuel Proskowetz jun. und Adolf Straka.

In Erwägung sollte die Möglichkeit der praktischen Verwendung der Musikinstrumente gezogen werden, die sich in den Sammlungen des mährischen Landesmuseums befinden, natürlich nicht nur aus der Provenienz Kvasice. Sie könnten im Konzertwesen neu erklingen und somit müssten sie nicht mehr nur stumme Museumsexponate bleiben. Damit die Instrumente für diese Zwecke verwendet werden könnten, müssten sie jedoch von einem Restaurator professionell repariert werden, denn keiner der Instrumente aus Kvasice ist derzeit im Stande, gespielt zu werden. Für die Restaurierung der ausgewählten Musikinstrumente wäre es angebracht, ein der Projekte von GA ČR ${ }^{35}$ zu nutzen.

Als Inspiration kann ein Konzert-Zyklus von der Gesellschaft der Musikfreunde in Wien unter den Namen „Nun klingen sie wieder" dienen. Hier geht es um Nutzung historischer Musikinstrumente aus den Sammlungen der Gesellschaft. In dieser Saison wurden sie beispielsweise anlässlich der Feierlichkeiten des 300. Geburtstags von Kaiserin

35 GA ČR - Grant-Agentur der Tschechischen Republik. 
Maria Theresa gespielt. Detailliert berichtet darüber der Direktor der GMF Otto Biba in seinem Artikel Altes, neu gehört. ${ }^{36}$

Übersetzt von Helena Kramářová

\section{Anhang}

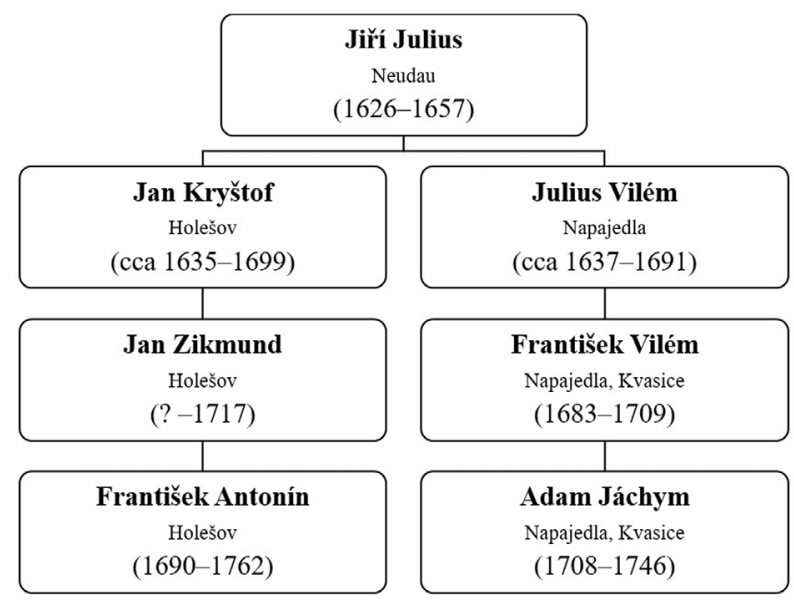

Abbildung 1

Ein Fragment des Stammbaums des Hauses von Rottal stellt das Verhältnis von Franz Anton und Adam Joachim von Rottal dar.

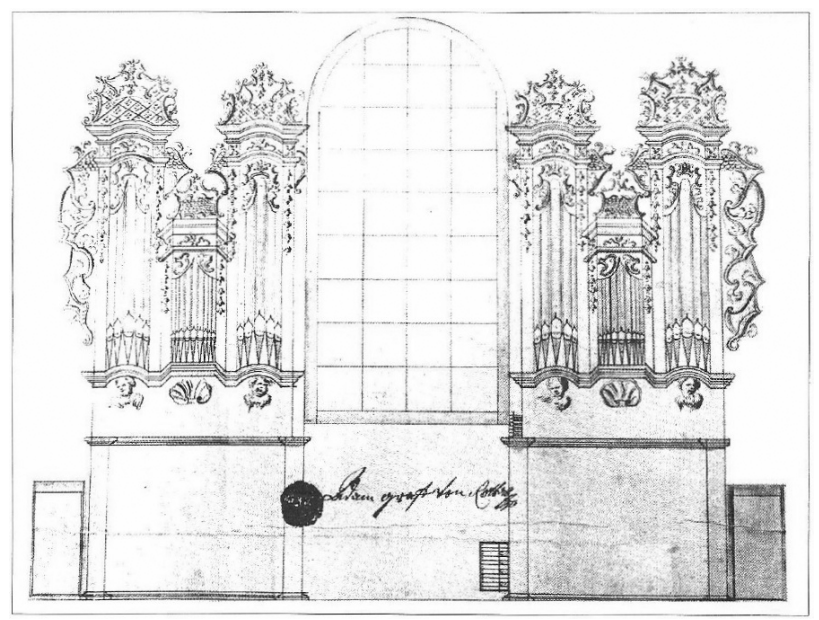

Abbildung 2

36 BIBA, Otto. Altes, neu gehört. Nun klingen sie wieder. In Magazin der Gesellschaft der Musikfreunde in Wien. Wien, April 2017, S. 1-3. 
Eine Kopie des Orgelentwurfs aus Kvasice vom Orgelbauer Adam König vom Jahr 1739.

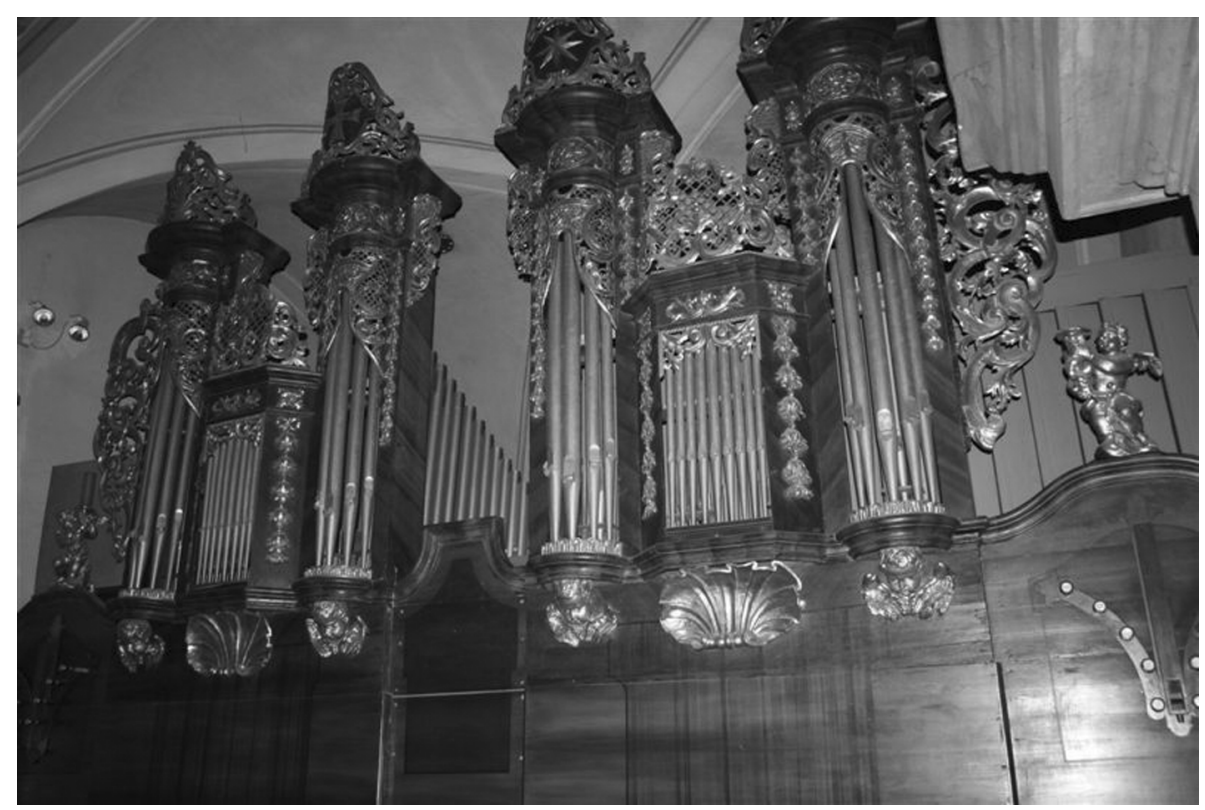

Abbildung 3

Der Orgelprospekt in der Kirche St. Margarethen in Jaroměřice nad Rokytnou (Aktueller Zustand).

\section{Bibliography}

\section{Sources}

SOkA Kroměříž. Farni fond Kvasice. Sign. Bj-20.

\section{Literature}

BEARE, Charles. Seraphin, Sanctus. [online], In Grove Music Online, [cit. 2017-08-25], Zugänglich unter: 〈http://www.oxfordmusiconline.com/subscriber/article/grove/music/25445>.

BIBA, Otto. Altes, neu gehört. Nun klingen sie wieder. In Magazin der Gesellschaft der Musikfreunde in Wien. Wien, April 2017.

DOSOUDIL, Václav. Katolická farnost Kvasice 2003. In Kvasické noviny, Kvasice. 3/2003.

Jahresbericht der Direktion für das 103. Vereinsjahr (1914/15) 1. Juli 1914 bis 30. Juni 1915. Erstattet in der ordentlichen Generalversammlung am 16. Dezember 1915. Wien 1915.

Jahresbericht der Direktion für das 106. Vereinsjahr (1917/18) 1. Juli 1917 bis 30. Juni 1918. Erstattet in der ordentlichen Generalversammlung am 19. Dezember 1918. Wien 1918. 
JALOVEC, Karel. Češti houslaři. Státní nakladatelství krásné literatury, hudby a umění Praha, 1959.

JALOVEC, Karel. Italšti houslaři. Orbis Praha, 1952.

JANEČEK, Pavel. Varhany na Kroměr $̌ z ̌ s k u$. Bachelorarbeit, Masaryk-Universität, Philosophische Fakultät, Institut für Musikwissenschaft, Brno 2004, Betreuer der Arbeit Stanislav Tesař.

SEHNAL, Jiří. Barokni varhanářstvi na Moravě. Díl. 1. Varhanáři. Muzejní a vlastivědná společnost v Brně, 2003. $171 \mathrm{~S}$.

SEHNAL, Jiří. Barokni varhanářstvi na Moravě. Dil. 2. Varhany. Muzejní a vlastivědná společnost v Brně, 2004. $294 \mathrm{~S}$.

SENN, Walter - ROY, Karl. Stainer, Jacob. [online]. In Grove Music Online. [Cit. 2017-08-25]. Zugänglich unter: 〈http://www.oxfordmusiconline.com/subscriber/article/grove/music/26528>.

STRAKOVÁ, Theodora. Kvasický inventář z r. 1757. In Časopis Moravského musea. roč. 38, Brno, 1953. S. 105-149. 
